\title{
Investigating the role of opiorphin genes in prostate cancer and the possible genetic mechanisms by which they modulate tumor growth and androgen- sensitivity.
}

\section{Amarnath Mukherjee}

Yeshiva University Albert Einstein College of Medicine

\section{Augene Park}

Yeshiva University Albert Einstein College of Medicine

\section{Li Wang}

Yeshiva University Albert Einstein College of Medicine

Kelvin Paul Davies ( $\sim$ kelvin.davies@einsteinmed.org)

Albert Einstein College of Medicine https://orcid.org/0000-0002-0201-9210

\section{Research article}

Keywords: Prostate Cancer, opiorphin, ProL1, hSMR3A, hSMR3B, xenograft, hypoxia, androgen-sensitivity

Posted Date: September 28th, 2020

DOI: https://doi.org/10.21203/rs.3.rs-30262/v2

License: (c) (i) This work is licensed under a Creative Commons Attribution 4.0 International License.

Read Full License 


\section{Abstract}

Background The opiorphin family of genes (represented in humans by ProL 1, hSMR3A and hSMR3B) encode peptides which act as potent neutral endopeptidase (NEP) inhibitors. Because modulated NEP activity is associated with cancer development it has been suggested that dysregulated opiorphin expression may be involved in oncogenesis and recent reports have associated upregulated opiorphin gene expression with breast and oropharyngeal cancer. These observations prompted the present studies to determine if opiorphin genes play a role in prostate cancer (PrCa).

Methods Publicly available data bases were screened for evidence associating opiorphin gene expression with PrCa and the findings confirmed using PrCa tissue arrays. Androgen-insensitive (PC3) and -sensitive (LNCaP) PrCa cells were engineered to overexpress ProL 1, referred to as LNCaP-ProL1+ and PC3-ProL1+, respectively. Xenografted tumor growth of ProL1-overexpressing and parental cell-lines were compared in male, castrated-male and female nude mice. Changes in global gene expression resulting from overexpression of ProL 1 in these cell-lines was determined by RNA-Seq.

Results Publicly available datasets supported an association between overexpression of opiorphin genes and $\mathrm{PrCa}$, which was confirmed using tissue arrays. Xenografted tumors derived from PC3-ProL $1+$ had an initial growth advantage over parent cell-lines in male mice, although at later time points there was no difference. Xenografted tumors derived from LNCaP-ProL1+ were able to grow in castrated male mice (in contrast to the parent cell-lines), and had impaired growth in female mice. Global gene expression analysis demonstrated that overexpression of ProL 1 causes modulated expression of genes involved in signaling, angiogenesis and steroid response pathways.

Conclusions This is the first report associating upregulated opiorphin gene expression and $\mathrm{PrCa}$. Xengrafted tumors derived from the androgen-sensitive LNCaP cell-line engineered to overexpress ProL 1 exhibit a more androgen-insensitive phenotype. Previous reports demonstrate opiorphins act as master regulators of the hypoxic response in smooth muscle cells. RNAseq data not only supports a similar role for ProL 1 in $\mathrm{PrCa}$ (regulating genes involved in angiogenesis), but in addition, regulating genes involved in steroid response. Therefore, opiorphins may play a role in PrCa development by activating pathways that overcome the hypoxic environment of the developing tumor and promote the development of androgen-insensitivity.

\section{Background}

Prostate cancer (PrCa) is the most prevalent of all cancers in men in the United States and the second most common cause of all cancer deaths. Despite high long-term survival rates with localized PrCa, metastatic PrCa remains largely incurable, even after intensive multimodal therapy [1]. Identifying novel molecular markers of $\mathrm{PrCa}$ and their involvement in genetic mechanisms leading to tumor growth and progression may lead to new therapeutic strategies to prevent and treat PrCa. Based on recent reports 
that dysregulated expression of opiorphin-encoding genes are associated with some cancers, we initiated the present studies to determine if they play a role in PrCa.

In humans, the opiorphin family genes are represented by ProL 1 , hSMR3A and $h S M R 3 B$, which are highly homologous genes that have neighboring genomic locations on chromosome 4 (cytogenetic location: 4q13.3) and have coregulated expression. Of the family of opiorphin-encoding genes, ProL 1 has been the most studied in the determination of their molecular and biochemical functions. The genes encode preproteins that are post-translationally processed to generate a bioactive pentapeptide, opiorphin, which acts as a potent endogenous neutral endopeptidase (NEP) inhibitor [2]. In addition to their activity as NEP inhibitors, members of opiorphin-encoding gene family have also been shown to play a role in the hypoxic response. For example, prior work from our laboratory has demonstrated that the rat homologue of ProL 1, vcsa 1, is upregulated in corporal smooth muscle cells in response to hypoxia, where it acts as a "master regulator" of the hypoxic response, regulating the expression of hypoxia-inducible factor $1 \mathrm{~A}$ (HIF1A) and vascular endothelial growth factor A (VEGFA) [3].

Opiorphins have been implicated in a wide-range of physiologic processes because of their biochemical activity as NEP inhibitors, which can potentially modulate the activity of peptide regulated signaling pathways. For example, early studies searching for a physiologic function demonstrated injection of supraphysiologic levels of opiorphin into the central nervous system of rodents had analgesic and antidepressive effects (hence the name "opiorphin" [4-8]). Subsequent studies demonstrated a role for opiorphin in regulating smooth muscle tone and through this activity a physiological role in both vascular and gastrointestinal tract biology [3, 9-21].

Because changes in NEP activity are associated with cancer development, a review article published in 2015 suggested that since opiorphin has biochemical activity as an NEP inhibitor, it may play a role in cancer [22]. This has been supported by several recent reports associating dysregulated expression opiorphin-encoding genes with cancer. Upregulated ProL 1 expression was observed in invasive multifocal/multicentric compared to unifocal breast cancer [23] and increased $h S M R 3 A$ expression was associated with unfavorable survival outcomes following surgery for oropharyngeal squamous cell carcinoma [24, 25]. In addition, in a meta-analysis using a rank aggregation approach to identify changes in gene expression in common between different cancer types, ProL 1 was included in the aggregated list of top-50 genes [26]. However, with the exception of a brief conference report from our laboratory [27], there are no published reports associating dysregulated expression of opiorphin-encoding genes with PrCa.

The activity of opiorphin as a master regulator of the hypoxic response [3] may also contribute to a role in the development and progression of PrCa. Since the metabolic demands of growing tumors creates a growth limiting hypoxic microenvironment [28-30] this might be expected to result in upregulation of opiorphin expression. Based on prior studies [3] the overexpression of opiorphin would in turn activate genetic pathways allowing the tumor to overcome the hypoxic barrier and continue to grow. In addition, 
given reports suggesting hypoxia may be a driver of malignant progression [31], ProL 1 might also be a factor in development of castration-resistant/androgen-insensitive PrCa.

In the present report we demonstrate that upregulated expression of opiorphins are associated with human $\mathrm{PrCa}$ and confirmed that ProL 1 overexpression modulates the growth and androgen-sensitivity of mouse xenografted PrCa tumors. Global gene expression analysis on the effects of overexpressing ProL 1 in PrCa cell-lines suggested possible genetic mechanisms by which ProL 1 modulates tumor growth and androgen-sensitivity consistent with its known biochemical and functional activities as an NEP inhibitor and master regulator of the hypoxic response.

\section{Methods}

\section{Identification of upregulated ProL 1 expression in prostate cancer}

An initial screen was conducted of publicly available gene expression datasets on the Gene Expression Omnibus (GEO) profiles database at NCBI [32] to determine if there was prior but unregognized evidence of an association between modulated expression of opiorphin encoding genes and PrCa. Using the search term "prostate cancer" identified 79 datasets. However, only 6 of these datasetss allowed both gene expression comparison between benign and cancerous prostate cancer tissue and included the opiorphin-encoding genes ProL $1, h S M R 3 A$ or $h S M R 3 B$. These 6 datasets were analyzed using the NCBI online data analysis tool (GEO2R) to compare expression of ProL 1 (referred to as OPRPNis this datasets), $h S M R 3 A$ and $h S M R 3 B$ between cancerous and non-cancerous prostate tissue. A change in expression of the a gene interest was considered significant if compared to the control group the gene had a Log2FoldChange of $>0.56$ or $<-0.56$ (i.e. a 1.5 -fold change) with a $p$-value $<0.05$. To provide context on how this analysis might also provide evidence of an association between $\mathrm{PrCa}$ and genes previously identified as markers of PrCa development and progression [33], we also screened the same 6 datasets to identify if PrCa was associated with changed expression of androgen receptor $(A R)$, breast cancer type 1 susceptibility protein (BRCA1), breast cancer type 2 susceptibility protein (BRCA2), cyclin-dependent kinase inhibitor N1B (CDKN1B), checkpoint kinase 2 (CHEK2), cytochrome P450 1B1 (CYP1B1), zinc phosphodiesterase ELAC protein 2 (ELAC2), tumor protein p53 (TP53), phosphatase and tensin homolog (PTEN) and RAS.

In order to confirm the upregulation of ProL 1 in PrCa, a CDNA array from normal and cancerous prostate tissue was purchased from OriGene (HPRT103; TissueScanTM cDNA Arrays; Rockville, MD, USA). This array has 48 samples covering 9-normal and 18-Stage II, 19-III and 2-IV PrCa. Quantitive RT-PCR analysis of the ProL 1 transcripts was performed as described below.

\section{Cell-lines}

PC3 (NCI-PBCF-CRL1435 (PC-3)/ATCC ${ }^{\circledR}$ CRL-1435 $5^{\text {TM}}$ ) and LNCaP clone FGC (NCI-PBCF-CRL1740 (LNCaP Clone FGC)/ATCC ${ }^{\circledR}$ CRL-1740 $0^{\mathrm{T}}$; hereafter termed LNCaP) PrCa cell-lines were obtained from the American Type Culture Collection (ATCC, Rockville, MD), and maintained in Roswell Park Memorial Institute (RPMI) 
1640 medium (Invitrogen, Carlsbad, CA) with 10\% Fetal Calf Serum (Thermo Fisher Scientific, Waltham, MA), supplemented with $100 \mathrm{U} / \mathrm{ml}$ penicillin $\mathrm{G}$ and $100 \mathrm{ng} / \mathrm{ml}$ streptomycin (Invitrogen). All cell-lines were passaged at 2-3 day intervals on reaching 70\% confluency using a $0.25 \%$ Trypsin-EDTA solution (Thermo Fisher Scientific). Cell morphology and viability were monitored by microscopic observation and regular Mycoplasma testing was performed (Universal Mycoplasma Detection Kit; ATCC).

\section{Generation of ProL 1 overexpressing cell-lines}

PC3 and LNCaP cells (as described above) were transduced with commercially available human ProL 1 lentiviral particles (Origene Technologies, Rockville, MD) according to manufacturers' protocol. The transduced cell-lines were designated PC3-ProL1+ and LNCaP-ProL1+ and over-expression of ProL 1 confirmed by quantitive RT-PCR. RNA seq data indicated ProL 1 was overexpressed 5,995-fold in PC3ProL1+ (see Supplemental Table 1) and 28,560-fold in LNCaP-ProL1+ (Supplemental Table 2) compared to their respective parent cell-lines. The doubling-time of PC3-ProL1+ and LNCaP-ProL1+ cell lines in culture was not significantly different to their parent cell lines.

\section{Mice xenograft studies}

All animal studies were conducted in accordance with the Animal (Scientific Procedures) Act 1986 and approved by the Institutional Animal Care and Use Committee (IACUC) of Albert Einstein College of Medicine. Athymic nude (homozygous, nu/nu aged 8-12 weeks) male, castrated-male and female mice (Charles River Laboratories, Wilmigton, MA) were housed under standard pathogen-free conditions at a maximum of five mice per cage. Tumours were established by subcutaneous injection on the upper left flank of nude mice randomly ascribed to groups that received PC3, PC3-ProL1+, LNCaP or LNCaP-ProL1+ cells in a 1:1 mixture of Matrigel (Corning Life Science, Teterboro, NJ) and HBSS using a 27-gauge needle. For PC3 and PC3-ProL1+ cell-lines, $1.5 \times 10^{6}$ cells, and for LNCaP and LNCaP-ProL1+ cell-lines, $2.5 \times 10^{6}$ cells were injected. The numbers of animals in each group are described in the Figure legends. Tumors were measured twice a week using Vernier Calipers and volume $\left(\mathrm{mm}^{3}\right)$ calculated by length $x$ width $\times$ height $\times 0.52$. When tumors reached a diameter $>1 \mathrm{~cm}$ mice were euthanized through inhalation of $\mathrm{CO}_{2}$ to effect.

\section{RNA Isolation}

To prepare total RNA to be used for quantitive-RT-PCR or RNA-seq analysis from in vitro experimental samples (PC3, PC3-ProL1+, LNCaP and LNCaP cell lines grown in culture), RNA was isolated with the RNeasy Plus Mini Kit (Qiagen, Hilden, Germany) following the manufacturers' protocol. To prepare total RNA from xenografted tumors for quantitive-RT-PCR analysis, tumors were first flash-frozen in liquid nitrogen and then ground to a homogenate using a pestle and mortar. RNA was isolated from $30 \mathrm{mg}$ of the homogenate using a combination of the TRIzol (Invitrogen) method and the RNeasy Plus Mini Kit (Qiagen).

\section{Quantitative RT-PCR}


Generation of cDNA was achieved using the SuperScript III First-Strand Synthesis System (Invitrogen) using $1 \mu \mathrm{g}$ total RNA as the starting material. For PCR analysis, samples were prepared in a $25 \mu \mathrm{L}$ reaction mix containing the PowerUp SYBR Green PCR Master Mix (Thermo Fisher). Real-time PCR was performed by the Applied Biosystems 7300 Real Time PCR System (Thermo Fisher) using the standard cycling mode recommended by the PowerUp SYBR protocol $\left(50^{\circ} \mathrm{C}\right.$ for 2 minutes, $95^{\circ} \mathrm{C}$ for 2 minutes, and then 40 cycles of $95^{\circ} \mathrm{C}$ for 15 seconds along with $60^{\circ} \mathrm{C}$ for 1 minute). Commercially available primers were obtained from Qiagen (RPL19, cat. \# PCRPPH18637A, ProL1 cat. \# PPH10360A and VEGFA cat. \# PPH00251C). Quantitative RT-PCR was performed in triplicate for each sample. Data was analyzed using the $\Delta \Delta \mathrm{Ct}$ method, with gene expression normalized to 60S ribosomal protein L19 (RPL19) and expressed as the average fold-change relative to controls.

\section{RNA-sequencing (RNA-seq)}

Prior to RNA-seq, RNA samples were treated with RNase-free DNase I and then quantified using RiboGreen (Thermo Fisher Scientific, Waltham, MA, USA). RNA integrity number (RIN) was assessed with an Agilent 2100 Bioanalyzer (Agilent, Santa Clara, CA, USA) to ensure each sample had a RIN $\geq 8$. RNA libraries were prepared using the Illumina TruSeq Stranded Total RNA kit, with Ribosomal depletion (Illumina, San Diego, CA, USA). Each library was sequenced using the Illumina HiSeq2500 platform and generated 125 nt paired-end $(P E)$ reads at either 30 million or 60 million reads per sample. RNA-seq analysis of gene expression for each cell-line was performed in triplicate (each sample prepared separately). [Raw sequencing datasets will be deposited in the Sequence Read Archive of NCBI (http://www.ncbi.nlm.nih.gov/sra) upon acceptance of this publication with accession number included here)]

Bioinformatics analysisThe reads were aligned with STAR (version 2.4.0c) [34] and genes annotated in Gencode v18 were quantified with featureCounts (v1.4.3-p1)[35]. Normalization and differential expression analyses were performed with the Bioconductor package DESeq2 [36]. Differentially expressed genes were assessed using a two-sided t-test and fold-change on log-transformed expression values. All samples were median centered. Cut-off criteria for differentially expressed genes was $>1$ Log2FoldChange or $<-1$ Log2FoldChange in gene expression with a $p$-value $<0.01$.

Gene ontology (GO) annotation analysis of differentially expressed genes was performed using online analysis tools available from the database for annotation, visualization and integrated discovery (DAVID, vers 6.8, Homo sapien GOTERM_GO_Direct database [37, 38]), the Gene Ontology Consortium (GOC, Homo sapien, biological function database $[39,40])$ and the Kyoto Encyclopedia of Genes and Genomes database (KEGG, [41-43]).

\section{Statistical analyses}

Statistical analyses were performed using either Microsoft Excel (Microsoft, Seattle, WA) or Prism 8.2. (GraphPad Software, Inc., La Jolla, CA). To determine statistical significance of two group comparisons, 
unpaired, two-tailed t-tests were performed, and results reported in Tables and Figures. Error bars represent standard error or standard deviation of mean (as described in figure legends).

\section{Results}

\section{ProL 1 is overexpressed in prostate cancer}

Of the 6 datasets in the GEO database that were suitable for our analysis, 2 demonstrated a significant association between PrCa and overexpression of opiorphin-encoding genes (Table 1 and Supplemental Table 3). In comparison, using the same criteria used to identify significant changes in opiorphin gene expression (Log2FoldChage of $>0.56$ or $<-0.56$, i.e. a 1.5 -fold change, with a p-value $<0.05$ ) revealed that out of the group of genes previously identified as associated with PrCa, the most commonly overrepresented gene in this analysis was BRAC1 in 4/6 datasets. The remaining genes were represented in only 2/6 datasets. A less stringent analysis datasets (a LogFC of $>0.26$ or $<-0.26$ i.e. a 1.2-fold change with a p-value $<0.1$ ) associated PrCa with a change in expression of opiorphin genes in $3 / 6$ datasets, BRAC1 in 5/6 datasets, PTEN and CYP1B1 in 4/6 datasets, AR in 3/6 datasets and the remaining genes (BRCA2, CDKN1B, CHEK2, ELAC2, RAS AND TP53) in 2/6 datasets. Therefore, a search of publicly available datasets associating PrCa with changed expression of the opiorphin genes identified an association in a similar number of datasets when the same search was applied to several other genes previously identified as associated with PrCa.

Table 1. The publicly available datasets GSE55945 and GSE3325 were analyzed using the GEO2R online tool to compare expression of opiorphin encoding genes (ProL1, hSMR3A and hSMR3B) between cancerous and non-cancerous prostate tissue samples.

\section{Comparison}

Dataset GSE55945

\section{Gene}

Gene

-value

\begin{tabular}{|l|l|} 
logFC & $\begin{array}{c}\text { Fold } \\
\text { Change }\end{array}$ \\
\hline
\end{tabular}

\begin{tabular}{l|c|c|c|c|}
\hline $\begin{array}{l}\text { PrCa data compared to non-cancerous } \\
\text { state tissue. }\end{array}$ & ProL1(OPRN) & 0.0013 & 1.14 & 2.20 \\
\cline { 2 - 5 } $\begin{array}{l}\text { 3 fusion positive PrCa data compared to non- } \\
\text { cerous prostate tissue. }\end{array}$ & ProL1(OPRN) & 0.00097 & 1.17 & 2.25 \\
\cline { 2 - 5 } & hSMR3A & 0.026 & 0.38 & 1.30 \\
\hline $\begin{array}{l}\text { j fusion negative PrCa data compared to } \\
\text {-cancerous prostate tissue. }\end{array}$ & ProL1(OPRN) & 0.013 & 1.11 & 2.16 \\
\cline { 2 - 5 } & SMR3A & 0.012 & 0.64 & 1.56 \\
\hline
\end{tabular}

\section{Dataset GSE3325}

\begin{tabular}{|c|c|c|c|c|}
\hline $\begin{array}{l}\text { PrCa data compared to non-cancerous } \\
\text { state tissue. }\end{array}$ & hSMR3B & 0.000083 & 2.74 & 6.68 \\
\hline $\begin{array}{l}\text { alized PrCa data compared to non-cancerous } \\
\text { state tissue. }\end{array}$ & hSMR3B & 0.0035 & 2.3 & 4.92 \\
\hline $\begin{array}{lcccc}\text { astatic PrCa data } & \text { compared } & \text { to } & \text { non- } \\
\text { cerous prostate tissue. } & & & \\
\end{array}$ & hSMR3B & 0.00039 & 3.22 & 9.32 \\
\hline
\end{tabular}


DataSet GSE55945 was generated from a study aimed at identifying novel biomarkers and immunotherapy targets for PrCa [44] and contained 8 normal (benign) prostate tissue samples and 13 prostate cancer tissues (sub-divided into ERG fusion positive ( $\mathrm{N}=6)$ and ERG fusion negative ( $\mathrm{N}=7$ ) $\mathrm{PrCa}$ ). As shown in Table 1, comparing the 8 normal prostate tissue samples and 13 prostate cancer tissues and using a p-value of $<0.05$ to define significance, PrCa was associated with overexpression of both ProL 1 and $h S M R 3 A$. When the group of prostate cancer tissues was sub-divided into ERG fusion positive and ERG fusion negative PrCa, there was a greater significance and fold-increase of ProL 1 expression in ERG fusion positive PrCa tissue compared to non-cancerous prostate tissue (2.25-fold, p-value $\left.9.7 \times 10^{-4}\right)$ than when the same analysis was performed with ERG fusion negative PrCa tissue (2.16-fold, $p$-value 1.3x10 $\left.{ }^{2}\right)$.

Dataset GSE3325 [45] was generated in a study to identify the genetic signatures of metastatic progression and contained 6 samples from normal (benign) prostate tissue samples and 13 PrCa tissues (sub-divided into localized ( $\mathrm{N}=6)$ and metastatic $(\mathrm{N}=7) \mathrm{PrCa}$ ). As shown in Table 1, PrCa was associated with overexpression of $h S M R 3 B$, with a trend for greater overexpression with progression from localized to metastatic disease, from 5 -fold ( $p$-value 0.0035 ) to 9.32-fold, ( $p$-value 0.00038 ), respectively.

Given that ProL 1 has been the most intensily studied memebr of the opiorphin gene family, we focused on this gene as represetative of the opiorphin family. Figure $1 A$ and $B$ show the relative expression levels of Prol1 determined in a tissue cDNA array containing human non-cancerous and cancerous (at different stages) prostate tissue. The data from the tissue array demonstrates that ProL 1 is overexpressed in $\mathrm{PrCa}$ tissue (at a similar level of overexpression as observed in the dataset analysis) and has a trend for higher levels of expression as the cancer stage increased. Overall, these analysis suggest that PrCa is associated with overexpression of ProL 1.

\section{Xenografted tumors derived from PC3 cell show a tumor-environment, time-dependent increase in the expression of ProL1 and VEGFA}

The metabolic demands of developing tumors create a hypoxic microenvironment that must be overcome for continued growth [28-30]. Because of our prior published studies suggesting that opiorphins are upregulated in smooth muscle cells in response to hypoxia, and then act as "master regulators" of the hypoxic response through the regulation of genes involved in the hypoxic response (such as VEGFA) [3], we determined if the microenvironment of a xenografted tumor would induce genes (ProL 1 and VEGFA) involved in hypoxic response pathways.

The relative expression of ProL 1 and VEGFA was compared between PC3 cells (isolated from castrationresistant/androgen-insensitive PrCa), xengrafted tumors derived from this cell-line at 2- and 4-week timepoints, and cells isolated from the xengrafted tumors and maintained under normoxic cell culture conditions. The PC3-derived tumors showed a progressive and significant increase in both ProL 1 and VEGFA expression (Figure 2A and B, respectively), such that after 4-weeks, tumors had approximately a 
20-fold increase in ProL 1 expression, and approximately a 3.2-fold increase in VEGFA expression, compared to the levels of these genes in the parent PC3 cell-line. In cells isolated from these tumors and cutured under normoxic conditions, the expression both ProL 1 and VEGFA returned to levels that were not significantly different from the parent PC3 cells. Overall, these data suggest that the hypoxic tumor microenvironment results in overexpression of ProL 1 and VEGFA in PC3 tumor xenograft.

\section{Xenografted tumors derived from a PC3 cell-line engineered to over-express ProL1 (PC3-ProL1+) initially exhibit accelerated growth compared to its parent cell-line in male nude mice}

In order to determine if overexpression of ProL 1 might directly affect PrCa tumor growth we compared the growth of xenografted tumors in male and female nude mice derived from a PC3 cell-line genetically engineered to overexpress ProL 1 (PC3-ProL1+) with its parent cell-line (PC3). As can be seen in Figure 3A, in male nude mice at early stages of tumor detection (at 10- and 13- days post-implantation), there was a significantly greater rate of tumor growth in mice implanted with PC3-ProL1+ compared to PC3. However, at later time-points (from 17-days onwards) in the male mice, and at any time-point in female nude mice, there was no significant size difference in the tumors derived from PC3-ProL1+ or the parental PC3 cells (Figure 3B and C).

\section{Overexpression of ProL1 in PC3 cells modulates expression of genes associated with the hypoxia and steroid response pathways.}

In order to identify possible genetic mechanisms by which upregulated ProL 1 expression in PC3 cells might modulate tumor growth, we compared global gene expression between the PC3 and PC3-ProL1+ cells grown in culture. This analysis identified 1,698 differentially expressed genes between the two celllines (Supplemental Table 1) with ProL 1 being the most differentially expressed gene (5,995-fold, $p$-value $\left.2.82 \times 10^{-26}\right)$. To identify biological functions that may be regulated through overexpression of ProL 1 in PC3 cells, the list of differentially expressed genes (minus ProL 1 ) was submitted to the DAVID, GOC and KEGG databases, which identified 1,250, 1,366 and 480 unique genes within each database, respectively, which were then used for ontological analysis. The most significant, broadly defined, functional ontologic groups with over-representation of differentially expressed genes were involved in morphogenesis and signal transduction (Supplemental Tables 4 and 5). For example, there was over-representation of differentially expressed genes in the ontological groups "anatomical structure

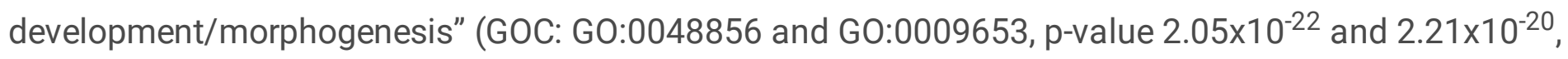
respectively, and more specifically "prostate gland morphogenesis" GOC: G0:0060512, p-value 6.39x10-4), "extracellular matrix organization" (DAVID: GO:0030198, 1.54x10-9), "signaling" (GOC: G0:0023052, p-

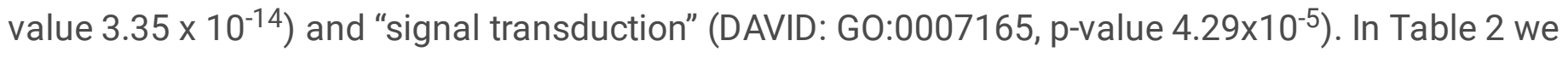
present the more defined ontologic groups where there was significant over-representation of differentially expressed genes. Both DAVID and GOC analysis identified differentially expressed genes in ontological groups involved in vascularization (such as genes involved in angiogenesis, blood vessel development, organ growth and morphogenesis) and also groups that function in hypoxia and direct modulation of blood flow (such as genes involved in over-coming hypoxia, vasodilation, blood vessel 
diameter and regulation of smooth muscle contraction). Analysis of the list of differentially expressed genes using the KEGG database identified the ontologic group "pathways in cancer" as having the greatest number of differentially expressed genes (41 genes, p-value 8.96×10-3) (Supplemental Table 6). Differentially expressed genes were also identified with significant overrepresentation in specific biochemical pathways, such as the P13K-Akt, VEGF and MAPK signaling pathways and steroid metabolic pathways (Table 2).

Table 2: Ontologic groups containing overrepresented differentially expressedgenes (DEG's) when ProL1 is overexpressed in PC3

\begin{tabular}{|c|c|c|c|c|c|c|}
\hline $\begin{array}{l}\text { Biological process/ } \\
\text { molecularpathway }\end{array}$ & $\begin{array}{l}\text { GO/hsa } \\
\text { identifier }\end{array}$ & Analysis & $\begin{array}{c}\text { Ref List } \\
\text { (GOC:20996) } \\
\text { (DAVD:16792) } \\
\text { (KEGG:6879) }\end{array}$ & $\begin{array}{c}\text { Prol1- } \\
\text { overexpression } \\
\text { list }\end{array}$ & $\begin{array}{l}\text { Fold- } \\
\text { enrichment. }\end{array}$ & P-value \\
\hline Angiogenesis & 0001525 & GOC & 315 & 56 & 2.65 & $9.33 \times 10^{-10}$ \\
\hline Bloodvessel development & 0001568 & GOC & 486 & 74 & 2.27 & $1.53 \times 10^{-9}$ \\
\hline Bloodvessel morphogenesis & 0048514 & GOC & 404 & 68 & 2.51 & $1.37 \times 10^{-10}$ \\
\hline Bloodcirculation & 0008015 & GOC & 386 & 63 & 2.44 & $2.55 \times 10^{-9}$ \\
\hline Circulatory system process & 0003013 & GOC & 394 & 77 & 2.18 & $3.9 \times 10^{-9}$ \\
\hline Angiogenesis & 0001525 & DAMD & 223 & 38 & 2.47 & $5.46 \times 10^{-11}$ \\
\hline Regulation of blood vessel diameter & 0097746 & GOC & 130 & 31 & 2.66 & $1.25 \times 10^{-5}$ \\
\hline Regulation of blood vessel size & 0050880 & GOC & 137 & 25 & 2.73 & $2.71 \times 10^{-5}$ \\
\hline $\begin{array}{l}\text { Positive regulation of animal organ } \\
\text { morphogenesis }\end{array}$ & 0110110 & GOC & 86 & 23 & 2.99 & $7.79 \times 10^{-5}$ \\
\hline Vasculogenesis & 0001570 & DAVD & 56 & 12 & 3.10 & $1.35 \times 10^{-3}$ \\
\hline Positive regulation of PI3K signaling & 0014068 & GOC & 85 & 15 & 2.64 & $1.4 \times 10^{-3}$ \\
\hline Regulation of blood pressure & 0045776 & DAVD & 30 & 13 & 2.90 & $1.47 \times 10^{-3}$ \\
\hline $\begin{array}{l}\text { Positive regulation of steroid metabolic } \\
\text { process }\end{array}$ & 0045940 & GOC & 28 & 8 & 4.27 & $1.47 \times 10^{-3}$ \\
\hline Response to hypoxia & 0001666 & DAMD & 172 & 22 & 1.85 & $7.7 \times 10^{-3}$ \\
\hline PI3K-Akt signaling pathway & 04151 & KEGG & 345 & 36 & 1.50 & $1.4 \times 10^{-2}$ \\
\hline VEGF signaling pathway & 04370 & KEGG & 61 & 10 & 2.35 & $2.4 \times 10^{-2}$ \\
\hline MAPK signaling pathway & 04010 & KEGG & 253 & 26 & 1.47 & $4.67 \times 10^{-2}$ \\
\hline Vasodilation & 0042311 & DAVD & 14 & 4 & 4.13 & $6.74 \times 10^{-2}$ \\
\hline
\end{tabular}

\section{Xenografted tumors derived from LNCaP cells engineered to over-express ProL1 (LNCaP-ProL1+) have gender-specific modulated growth compared to its parent cell-line.}

We expanded the studies described above for the androgen insensitive PrCa cell-line, PC3, to determine if upregulated ProL 1 expression might also play a role in tumor development in an androgen sensitive cellline $(\mathrm{LNCaP})$. We compared the growth between xenografted tumors derived from LNCaP cells engineered to over-express ProL 1 (LNCaP-ProL1+) and their parental cells in male, castrated-male and female nude mice (Figure 4). Although overexpression of ProL 1 in LNCaP cells did not affect tumor growth in male mice (Figure 4A) it significantly modulated tumor growth in both castrated-male and female mice (Figure 4B and C). As expected for an androgen sensitive cell-line, no tumors developed when LNCaP cells were xenografted into castrated-male mice (Figure 4B). Remarkably, although detection of tumors was delayed from 3 to 7 weeks, xenografted LNCaP-ProL1+ cells were able to develop tumors in castrated-male mice. In addition, in at least one of the 8 castrated-male mice xenografted with LNCaP-ProL1+ cells, several secondary tumors were observed. Similar to previous studies [46], xenografted tumors derived from LNCaP did develop tumors in female mice, albeit at a delayed time for detection compared to male nude mice. However, once LNCaP tumors are established, their growth rate is not significantly different 
between male and female mice. Interestingly, in female mice there was significantly impaired growth of tumors derived from LNCaP-ProL1+ compared to tumors derived from LNCaP (Figure 4C). Overall these observations suggest that overexpressing ProL 1 in the androgen sensitive LNCaP cell-line modulates the hormonal sensitivity of xenografted tumors towards a more androgen-insensitive phenotype.

\section{Overexpression of ProL1 in LNCaP cells modulates expression of genes associated with steroid metabolism and the androgen response}

In order to determine possible mechanisms for the difference in xenografted LNCaP tumor growth when ProL 1 is overexpressed, we compared global gene expression between the LNCaP-ProL $1+$ and LNCaP (its parent cell-line) growing in culture, identifying 1,110 differentially expressed genes (Supplemental Table 2). Of these 1, differentially expressed genes, 209 were in common with PC3 when ProL 1 was overexpressed (Supplemental Table 2). ProL 1 was the most differentially expressed gene (28,560-fold, pvalue $\left.24.36 \times 10^{-36}\right)$. To identify biological functions that may be regulated through overexpression of ProL 1 in LNCaP cells, the list of differentially expressed genes (minus ProL 1 ) was submitted to the DAVID, GOC and KEGG databases, identifying 665, 812 and 287 unique genes within each database, respectively, which were then used for ontological analysis. Although the analysis identifed fewer ontologic groups with significant overrepresentation of differentially expressed genes caused by ProL 1 overexpression in LNCaP compared to PC3 cells, many these ontologic groups were the same, or served similar functions (Supplemental Tables 7, 8 and 9). In common with PC3, ProL 1 overexpression regulated gene expression in ontological groups involved in morphogenesis and signal transduction (Supplemental Tables 7 and 8). For example, there was over-representation of genes in the ontological groups "anatomical structure development/morphogenesis" (GOC: GO:0048856/G0:0009653, p-value 2.76x108

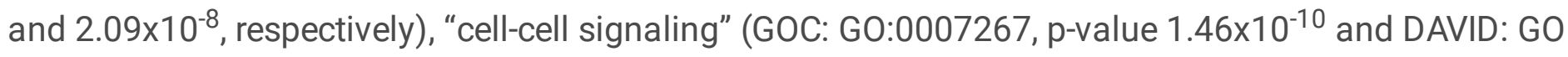

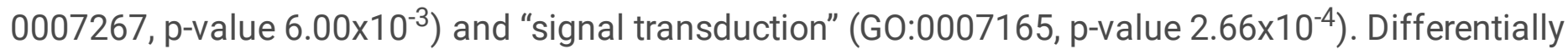
expressed genes were also overrepresented in more definded ontological groups potentially involved in vascularization (angiogenesis, circulatory system development and organ morphogenesis) and regulation of blood flow (smooth muscle contraction and blood pressure) (Table 3). Analysis for overrepresentation of differentially expressed genes in biochemical pathways using the KEGG database, also identified similar effects caused by overexpression of ProL 1 in PC3 and LNCaP cells (Supplemental Table 9). For example, overexpressing ProL 1 regulated genes that were significantly over-represented in the ontologic groups "pathways in cancer" ( $p$-value $5.97 \times 10^{-2}$ ), steroid metabolism and the regulation of MAPK signaling (Table 3). 
Table 3: Ontologic group s containing overrepresented differentially expres sed genes(DEG's) when ProL 1 is overexp res sed in LNCaP cells.

\begin{tabular}{|c|c|c|c|c|c|c|}
\hline $\begin{array}{l}\text { Biological process/ } \\
\text { molecular pathway }\end{array}$ & $\begin{array}{l}\text { GO/hsa } \\
\text { identifier }\end{array}$ & Anglys is & $\begin{array}{c}\text { Ref List } \\
\text { (GOC:20996) } \\
\text { (DAVID:16792) } \\
\text { (KEGG:6879) }\end{array}$ & $\begin{array}{c}\text { Proll- } \\
\text { overexpress ion } \\
\text { list }\end{array}$ & $\begin{array}{l}\text { Fold- } \\
\text { enrichment. }\end{array}$ & P-value \\
\hline An imal organ morphogenesis & 0009887 & GOC & 930 & 68 & 1.79 & $1.16 \times 10^{-5}$ \\
\hline Smooth muscle con traction & 0006939 & GOC & 51 & 10 & 4.8 & $1.20 \times 10^{-1}$ \\
\hline Circulatory system development & 0072359 & GOC & 832 & 59 & 1.7 & $1.60 \times 10^{-1}$ \\
\hline Blood circulation & 0008015 & GOC & 388 & 33 & 209 & $1.72 \times 10^{-}$ \\
\hline Stero id hom one biosynthesis & 00140 & KEGG & 58 & 8 & 3.31 & $9.75 \times 10^{-3}$ \\
\hline Angiogenesis & 0001525 & DAMD & 223 & 16 & 1.81 & 3. $19 \times 10^{-2}$ \\
\hline Po sitive regulation of MAPK cascade & 0043410 & DAMD & 81 & 8 & 2.49 & $4.10 \times 10^{-2}$ \\
\hline Po sitive regulation of blood pressure & 0045777 & DAMD & 20 & 4 & 5.05 & $4.25 \times 10^{-2}$ \\
\hline
\end{tabular}

However, there were differences in the regulation of specific genes related to the androgen response between LNCaP and PC3. Unlike PC3, where overexpression of ProL 1 caused a significant increase in expression of the androgen receptor gene (AR, 7.46-fold, p-value 3.16x10-197) and a decrease in expression of the estrogen receptor (ESR1, 2.7-fold reduced, p-value $3.38 \times 10^{-49}$ ), in LNCaP neither of these genes were changed in expression (Supplemental Table 1 and 2). In contrast, overexpression of ProL 1 in LNCaP reduced expression of the progesterone receptor gene (PGR, 2.17-fold reduced, p-value 4.41x10-3), whereas PGR expression was not changed in PC3 cells.

\section{Discussion}

This is the first report associating elevated expression levels of opiorphin-encoding genes with the development of human PrCa. An initial screen of publicly available databases identified an association between expression levels of the opiorphin-encoding genes and PrCa. As shown in Table 1 there is a much greater significance of elevated ProL 1 expression in ERG-fusion positive $\mathrm{PrCa}$ (which is generally considered to represent a more advanced stage PrCa [47]) compared to ERG-fusion negative PrCa (Table 1). There was overexpression of the ProL 1 homologue, $h S M R 3 B$, in localized PrCa compared to noncancerous prostate tissue (5-fold, p-value 0.0035$)$, with greater overexpression in metastatic PrCa compared to non-cancerous prostate tissue (9.32-fold, p-value 0.00038 ). Although only 2 of the 6 datasets publicly available on GEO associated an upregulation of opiorphin genes with cancer development, a similar search for an association with several generally well-accepted genes involved in PrCa revealed associations in similar numbers of datasets (Supplemental Table 3). In a meta-analysis of 20 microrarray data sets using a rank aggregation approach to identify changes in gene expression in common between different cancer types, the maximum number of lists that any gene appeared in was 3 [26]. In this study, of the aggregated list of top- 50 genes, 36 had been previously implicated in cancer (often multiple cancers) and the authors suggested that the other 14 genes "may contain some novel cancer genes that may deserve further scrutiny in the future". It is interesting to that ProL 1 , the gene encoding opiorphin, was one of these 14 genes.

The apparent low level representation in datasets of the differentially expressed opiorphin genes, as well as other markers of PrCa, may represent the highly heterogenous nature of samples used in gene expression studies [48]. Not only is PrCa in general highly heterogeneous in nature, but even in the same 
patient there are multiple distinct cancer foci at primary diagnosis. It has also been shown that many PrCa datasets have significant stromal contamination which has a tendency to mask epithelial gene expression across PrCa profiling studies [49]. Subsequently, PrCa tissue arrays confirmed that ProL 1 is significantly upregulated in $\mathrm{PrCa}$, with a trend for higher levels of overexpression with stage (Figure 1).

Our data (presented in Figure 2) suggests that overexpression of opiorphin genes is a response to the tumor microenvironment. Because our prior published studies have demonstrated that opiorphins are upregulated in smooth muscle cells in response to hypoxia [3], and that the metabolic demands of developing tumors create a hypoxic microenvironment [28-30], our hypothesis is that it is the hypoxic environment of the developing tumor that results in opiorphin overexpression.

We also demonstrate that engineered constitutive overexpression of ProL 1 modulates the growth of xenografted tumors. The effect on tumor growth caused by overexpression of ProL 1 is highly pronounced in LNCaP cells (isolated from castration-sensitive/androgen-sensitive PrCa) (Figure 4). Remarkably, in contrast to the parent cells, xenografted LNCaP-ProL $1+$ cells were able to develop tumors in castratedmale mice and in female mice overexpression of ProL 1 significantly impaired growth of tumors. In PC3 cells (a castration-resistant/ androgen-insensitive PrCa cell-line) although overexpression of ProL 1 increases the growth rate of xenografted tumors in nude male mice at early time-points there is no significant difference at later time points (Figure 3). These experiments provide strong evidence ProL 1 overexpression modulates the androgen sensitivity of tumors, such that the xenografted tumor exhibits a more androgen-insensitive phenotype.

Global analysis of the changes in gene expression caused by overexpression of ProL 1 in PC3 and LNCaP cells supports that it has a role both in the modulation of genetic pathways involved in both overcoming hypoxia and the development of androgen-insensitivity. Opiorphins have previously been shown to be directly involved in regulation of blood-flow to tissues through their modulation of smooth muscle tone $[11,14,18]$, and therefore their upregulation in tumors may directly contribute to overcoming the hypoxic barrier that develops in the growing tumor. In addition, overexpression of ProL 1 in both PC3 and LNCaP cells modulated expression of genes involved in angiogenesis and morphogensis (Table 2 and 3); the activation of these pathways would likely be involved in vascularization of the tumor and thereby also contribute to overcoming hypoxia.

Overexpression of ProL 1 also affected genes involved in steroid metabolism and response pathways in both LNCaP and PC3 (Table 2 and 3), which could potentially contribute to the modulation of androgen sensitivity. For example, overexpression of ProL 1 in $L N C a P$ reduced expression of the progesterone receptor gene (PGR). Activation of PGR is a positive modulator of cell division $[50,51]$ and the reduced expression of $P G R$ in LNCaP-ProL1 cells might therefore explain the negative effect on tumor growth in female mice. In PC3-ProL 1+ cells we observed a significant upregulation of the AR (7.46-fold, p-value 3.16x10-197, Supplemental Table 1 ). Increased activity of the AR could subsequently lead to the activation of secondary messengers involved in modulating the activity of other signaling pathways, such as the PI3K-Akt signaling and MAPK pathways which had significant over-representation of differentially

Page $13 / 22$ 
expressed genes in PC3-ProL1+ cells. The PI3K-Akt signaling pathway is considered one of the most commonly dysregulated pathways in PrCa with approximately $40 \%$ of early stage, and $70-100 \%$ of advanced stage PrCa, exhibiting dysregulation of this pathway, suggesting PI3K-Akt dysregulation is associated with PrCa progression [52, 53]

Overall, our data associates the development of PrCa with overexpression of opiorphin and suggests genetic pathways by which they contribute to tumor growth and development androgen-insensitivity. These mechanisms are summarized in Figure 5. The hypoxic barrier to growth developed when tumors reach about $2 \mathrm{~mm}^{3}$ would be expected to upregulate opiorphin gene expression. As master regulators of the hypoxic response, overexpression of opiorphin would both directly relax vascular smooth muscle cells neighboring the tumor, and activate pathways involved in the vascularization and regulation of blood supply to the tumor, allowing tumors to overcome the hypoxic barrier and PrCa growth. In addition, overexpression of ProL 1 in response to hypoxia may activate genes involved in steroid metabolism and androgen response, modulating the androgen sensitivity of the tumor and potentially driving the development of castration resistance/androgen-insensitivity.

\section{Conclusion}

In conclusion, this is the first report associating upregulated opiorphin gene expression and PrCa. Overexpression of ProL 1 in PrCa cell-lines modulates tumor growth and, in the androgen-sensitive LNCaP cell-line, xenografted tumors exhibit a more androgen-insensitive phenotype. Global analysis of the changes in gene expression caused by overexpression of ProL 1 in PC3 and LNCaP cells supports that it has a role both in the modulation of genetic pathways involved in both overcoming hypoxia and the development of androgen-insensitivity. Therefore, opiorphins may play a role in PrCa development by activating pathways that overcome the hypoxic environment of the developing tumor and modulate its androgen sensitivity. Strategies that target opiorphin expression may represent a novel strategy preventing the development and progression of PrCa.

\section{Abbreviations}

PrCa, Prostate Cancer; NEP, neutral endopeptidase; GEO, Gene Expression Omnibus; GO, gene ontology; DAVID, database for annotation, visualization and integrated discovery; GOC, Gene Ontology Consortium; KEGG, Kyoto Encyclopedia of Genes and Genomes; AR, androgen receptor; BRCA1, breast cancer type 1 susceptibility protein; BRCA2, breast cancer type 2 susceptibility protein; CDKN1B, cyclin-dependent kinase inhibitor N1B; CHEK2, checkpoint kinase 2; CYP1B1, cytochrome P450 1B1; ELAC2, zinc phosphodiesterase ELAC protein 2; TP53, tumor protein p53; PTEN, phosphatase and tensin homolog; ProL1 (aka OPRPN), proline rich, lacrimal 1 (human opiorphin encoding gene), hSMR3A/B, human submaxillary gland androgen regulated protein 3 , homolog $A$ (human opiorphin encoding gene)

\section{Declarations}




\section{Ethics approval and consent to participate}

All animal studies were conducted in accordance with the Animal (Scientific Procedures) Act 1986 and approved by the Institutional Animal Care and Use Committee (IACUC) of Albert Einstein College of Medicine.

\section{Consent for publication}

Not applicable.

\section{Availability of data and materials}

[Raw sequencing datasets will be deposited in the Sequence Read Archive of NCBI (http://www.ncbi.nlm.nih.gov/sra) upon acceptance of this publication with accession number included here)] .

\section{Competing interests}

The authors declare that they have no competing interests.

\section{Funding}

Funding for these studies were provided by NIH R01 DK107807 (PI: Kelvin P. Davies). The funders had no role in study design, data collection and analysis, decision to publish, or preparation of the manuscript.

\section{Authors' contributions}

"AM conducted, analyzed and interpreted data in manuscript and contributed to writing the manuscript. AP conducted, analyzed and interpreted data in manuscript and contributed to writing the manuscript. LW AP conducted and analyzed data in manuscript. KPD conceived of the study, analyzed and interpreted data in manuscript and was the major contributor to writing the manuscript. All authors read and approved the final manuscript

\section{Acknowledgements}

We thank Mark Schoenberg, MD, for his initial involvement in discussions in the study conception.

\section{Authors' information (optional).}

None.

\section{References}

1. Wang G, Zhao D, Spring DJ, DePinho RA: Genetics and biology of prostate cancer. Genes \& development 2018, 32(17-18):1105-1140. 
2. Wisner A, Dufour E, Messaoudi M, Nejdi A, Marcel A, Ungeheuer MN, Rougeot C: Human Opiorphin, a natural antinociceptive modulator of opioid-dependent pathways. Proc Natl Acad Sci U S A 2006, 103(47):17979-17984.

3. Fu S, Tar MT, Melman A, Davies KP: Opiorphin is a master regulator of the hypoxic response in corporal smooth muscle cells. FASEB journal : official publication of the Federation of American Societies for Experimental Biology 2014, 28(8):3633-3644.

4. Benyhe Z, Toth G, Wollemann M, Borsodi A, Helyes Z, Rougeot C, Benyhe S: Effects of synthetic analogues of human opiorphin on rat brain opioid receptors. Journal of physiology and pharmacology : an official journal of the Polish Physiological Society 2014, 65(4):525-530.

5. Toth F, Toth G, Benyhe S, Rougeot C, Wollemann M: Opiorphin highly improves the specific binding and affinity of MERF and MEGY to rat brain opioid receptors. Regulatory peptides 2012, 178(1-3):7175 .

6. Rosa M, Arsequell G, Rougeot C, Calle LP, Marcelo F, Pinto M, Centeno NB, Jimenez-Barbero J, Valencia G: Structure-activity relationship study of opiorphin, a human dual ectopeptidase inhibitor with antinociceptive properties. Journal of medicinal chemistry 2012, 55(3):1181-1188.

7. Yang QZ, Lu SS, Tian XZ, Yang AM, Ge WW, Chen Q: The antidepressant-like effect of human opiorphin via opioid-dependent pathways in mice. Neuroscience letters 2011, 489(2):131-135.

8. Javelot $\mathrm{H}$, Messaoudi M, Garnier S, Rougeot $\mathrm{C}$ : Human opiorphin is a naturally occurring antidepressant acting selectively on enkephalin-dependent delta-opioid pathways. Journal of physiology and pharmacology : an official journal of the Polish Physiological Society 2010, 61(3):355-362.

9. Chua RG, Calenda G, Zhang X, Siragusa J, Tong Y, Tar M, Aydin M, DiSanto ME, Melman A, Davies KP: Testosterone regulates erectile function and Vcsa1 expression in the corpora of rats. Molecular and cellular endocrinology 2009, 303(1-2):67-73.

10. Calenda G, Tong Y, Tar M, Lowe D, Siragusa J, Melman A, Davies KP: Vcsa1 Acts as a Marker of Erectile Function Recovery After Gene Therapeutic and Pharmacological Interventions. J Uro/ 2009.

11. Tong $Y$, Tar M, Melman A, Davies K: The opiorphin gene (ProL1) and its homologues function in erectile physiology. BJU Int 2008, 102(6):736-740.

12. Davies KP, Melman A: Markers of erectile dysfunction. Indian journal of urology : IJU : journal of the Urological Society of India 2008, 24(3):320-328.

13. Tong $Y$, Tar M, Monrose V, DiSanto M, Melman A, Davies KP: hSMR3A as a marker for patients with erectile dysfunction. J Urol 2007, 178(1):338-343.

14. Davies KP, Tar M, Rougeot C, Melman A: Sialorphin (the mature peptide product of Vcsa1) relaxes corporal smooth muscle tissue and increases erectile function in the ageing rat. BJU Int 2007, 99(2):431-435.

15. Tong Y, Tar M, Davelman F, Christ G, Melman A, Davies KP: Variable coding sequence protein A1 as a marker for erectile dysfunction. BJU Int 2006, 98(2):396-401. 
16. Davies KP, Tong Y, Tar M, Lowe D, Melman A: Vcsa1 (SMR1) as a molecular marker for treatment of erectile dysfunction. J Sex Med 2006, 3(Suppl 5):414.

17. Tian XZ, Chen Y, Bai L, Luo P, Du XJ, Chen Q, Tian XM: Effects and underlying mechanisms of human opiorphin on cardiovascular activity in anesthetized rats. European journal of pharmacology 2014, 749C:32-38.

18. Calenda G, Tong Y, Kanika ND, Tar MT, Suadicani SO, Zhang X, Melman A, Rougeot C, Davies KP: Reversal of diabetic vasculopathy in a rat model of type 1 diabetes by opiorphin-related peptides. American journal of physiology Heart and circulatory physiology 2011, 301(4):H1353-1359.

19. Kanika ND, Tar M, Tong Y, Kuppam DS, Melman A, Davies KP: The mechanism of opiorphin-induced experimental priapism in rats involves activation of the polyamine synthetic pathway. American journal of physiology Cell physiology 2009, 297(4):C916-927.

20. Tian XZ, Chen J, Xiong W, He T, Chen Q: Effects and underlying mechanisms of human opiorphin on colonic motility and nociception in mice. Peptides 2009, 30(7):1348-1354.

21. Kamysz E, Sałaga M, Sobczak M, Kamysz W, Fichna J: Characterization of the effects of opiorphin and sialorphin and their analogs substituted in position 1 with pyroglutamic acid on motility in the mouse ileum. J Pept Sci 2013, 19(3):166-172.

22. Mizerska-Dudka M, Kandefer-Szerszeń M: Opioids, Neutral Endopeptidase, its Inhibitors and Cancer: Is There a Relationship among them? Arch Immunol Ther Exp (Warsz) 2015, 63(3):197-205.

23. Lang Z, Wu Y, Pan X, Qu G, Zhang T: Study of differential gene expression between invasive multifocal/ multicentric and unifocal breast cancer. Journal of BUON : official journal of the Balkan Union of Oncology 2018, 23(1):134-142.

24. Koffler J, Holzinger D, Sanhueza GA, Flechtenmacher C, Zaoui K, Lahrmann B, Grabe N, Plinkert PK, Hess J: Submaxillary gland androgen-regulated protein $3 A$ expression is an unfavorable risk factor for the survival of oropharyngeal squamous cell carcinoma patients after surgery. European archives of oto-rhino-laryngology : official journal of the European Federation of Oto-Rhino-Laryngological Societies 2013, 270(4):1493-1500.

25. Grunow J, Rong C, Hischmann J, Zaoui K, Flechtenmacher C, Weber KJ, Plinkert P, Hess J: Regulation of submaxillary gland androgen-regulated protein 3A via estrogen receptor 2 in radioresistant head and neck squamous cell carcinoma cells. Journal of experimental \& clinical cancer research : CR 2017, 36(1):25.

26. Pihur V, Datta S, Datta S: Finding common genes in multiple cancer types through meta-analysis of microarray experiments: a rank aggregation approach. Genomics 2008, 92(6):400-403.

27. Mukherjee A, Wang L, Schoenberg M, Davies K: Upregulation of opiorphin is associated with prostate cancer, a potential novel therapeutic target. Urology 2017, 197(4):e1167-e1168.

28. Carmeliet P, Jain RK: Angiogenesis in cancer and other diseases. Nature 2000, 407(6801):249-257.

29. Fox WD, Higgins B, Maiese KM, Drobnjak M, Cordon-Cardo C, Scher HI, Agus DB: Antibody to vascular endothelial growth factor slows growth of an androgen-independent xenograft model of prostate 
cancer. Clinical cancer research : an official journal of the American Association for Cancer Research 2002, 8(10):3226-3231.

30. Chung LW, Baseman A, Assikis V, Zhau HE: Molecular insights into prostate cancer progression: the missing link of tumor microenvironment. J Urol 2005, 173(1):10-20.

31. Rudolfsson SH, Bergh A: Hypoxia drives prostate tumour progression and impairs the effectiveness of therapy, but can also promote cell death and serve as a therapeutic target. Expert opinion on therapeutic targets 2009, 13(2):219-225.

32. Barrett T, Wilhite SE, Ledoux P, Evangelista C, Kim IF, Tomashevsky M, Marshall KA, Phillippy KH, Sherman PM, Holko M et al: NCBI GEO: archive for functional genomics data sets-update. Nucleic acids research 2013, 41(Database issue):D991-995.

33. Fu S, Davies KP: Opiorphin-dependent upregulation of CD73 (a key enzyme in the adenosine signaling pathway) in corporal smooth muscle cells exposed to hypoxic conditions and in corporal tissue in pre-priapic sickle cell mice. International journal of impotence research 2015, 27(4):140-145.

34. Dobin A, Davis CA, Schlesinger F, Drenkow J, Zaleski C, Jha S, Batut P, Chaisson M, Gingeras TR: STAR: ultrafast universal RNA-seq aligner. Bioinformatics 2013, 29(1):15-21.

35. Liao Y, Smyth GK, Shi W: featureCounts: an efficient general purpose program for assigning sequence reads to genomic features. Bioinformatics 2014, 30(7):923-930.

36. Love MI, Huber W, Anders S: Moderated estimation of fold change and dispersion for RNA-seq data with DESeq2. Genome biology 2014, 15(12):550.

37. Huang da W, Sherman BT, Lempicki RA: Systematic and integrative analysis of large gene lists using DAVID bioinformatics resources. Nature protocols 2009, 4(1):44-57.

38. Huang da W, Sherman BT, Lempicki RA: Bioinformatics enrichment tools: paths toward the comprehensive functional analysis of large gene lists. Nucleic acids research 2009, 37(1):1-13.

39. Ashburner M, Ball CA, Blake JA, Botstein D, Butler H, Cherry JM, Davis AP, Dolinski K, Dwight SS, Eppig JT et al: Gene ontology: tool for the unification of biology. The Gene Ontology Consortium. Nature genetics 2000, 25(1):25-29.

40. The Gene Ontology C: The Gene Ontology Resource: 20 years and still GOing strong. Nucleic acids research 2019, 47(D1):D330-D338.

41. Kanehisa M, Furumichi M, Tanabe M, Sato Y, Morishima K: KEGG: new perspectives on genomes, pathways, diseases and drugs. Nucleic acids research 2017, 45(D1):D353-D361.

42. Kanehisa M, Sato Y, Furumichi M, Morishima K, Tanabe M: New approach for understanding genome variations in KEGG. Nucleic acids research 2019, 47(D1):D590-D595.

43. Yamamoto S, Teng W, Kakiuchi T, Tsukada H: Disturbance of cerebral blood flow autoregulation in hypertension is attributable to ischaemia following subarachnoid haemorrhage in rats: A PET study. Acta neurochirurgica 1999, 141(11):1213-1219.

44. Arredouani MS, Lu B, Bhasin M, Eljanne M, Yue W, Mosquera JM, Bubley GJ, Li V, Rubin MA, Libermann TA et al: Identification of the transcription factor single-minded homologue 2 as a 
potential biomarker and immunotherapy target in prostate cancer. Clinical cancer research : an official journal of the American Association for Cancer Research 2009, 15(18):5794-5802.

45. Varambally S, Yu J, Laxman B, Rhodes DR, Mehra R, Tomlins SA, Shah RB, Chandran U, Monzon FA, Becich $\mathrm{MJ}$ et al: Integrative genomic and proteomic analysis of prostate cancer reveals signatures of metastatic progression. Cancer cel/ 2005, 8(5):393-406.

46. Horoszewicz JS, Leong SS, Kawinski E, Karr JP, Rosenthal H, Chu TM, Mirand EA, Murphy GP: LNCaP model of human prostatic carcinoma. Cancer research 1983, 43(4):1809-1818.

47. Song C, Chen H: Predictive significance of TMRPSS2-ERG fusion in prostate cancer: a meta-analysis. Cancer cell international 2018, 18:177.

48. Carm KT, Hoff AM, Bakken AC, Axcrona U, Axcrona K, Lothe RA, Skotheim RI, Løvf M: Interfocal heterogeneity challenges the clinical usefulness of molecular classification of primary prostate cancer. Sci Rep 2019, 9(1):13579.

49. Tomlins SA, Mehra R, Rhodes DR, Cao X, Wang L, Dhanasekaran SM, Kalyana-Sundaram S, Wei JT, Rubin MA, Pienta KJ et al: Integrative molecular concept modeling of prostate cancer progression. Nature genetics 2007, 39(1):41-51.

50. Bonkhoff $\mathrm{H}$ : Estrogen receptor signaling in prostate cancer: Implications for carcinogenesis and tumor progression. The Prostate 2018, 78(1):2-10.

51. Bonkhoff H, Fixemer T, Hunsicker I, Remberger K: Progesterone receptor expression in human prostate cancer: correlation with tumor progression. The Prostate 2001, 48(4):285-291.

52. Carver BS, Chapinski C, Wongvipat J, Hieronymus H, Chen Y, Chandarlapaty S, Arora VK, Le C, Koutcher $\mathrm{J}$, Scher $\mathrm{H}$ et al: Reciprocal feedback regulation of $\mathrm{PI} 3 \mathrm{~K}$ and androgen receptor signaling in PTEN-deficient prostate cancer. Cancer cell 2011, 19(5):575-586.

53. Edlind MP, Hsieh AC: PI3K-AKT-mTOR signaling in prostate cancer progression and androgen deprivation therapy resistance. Asian journal of andrology 2014, 16(3):378-386.

\section{Figures}
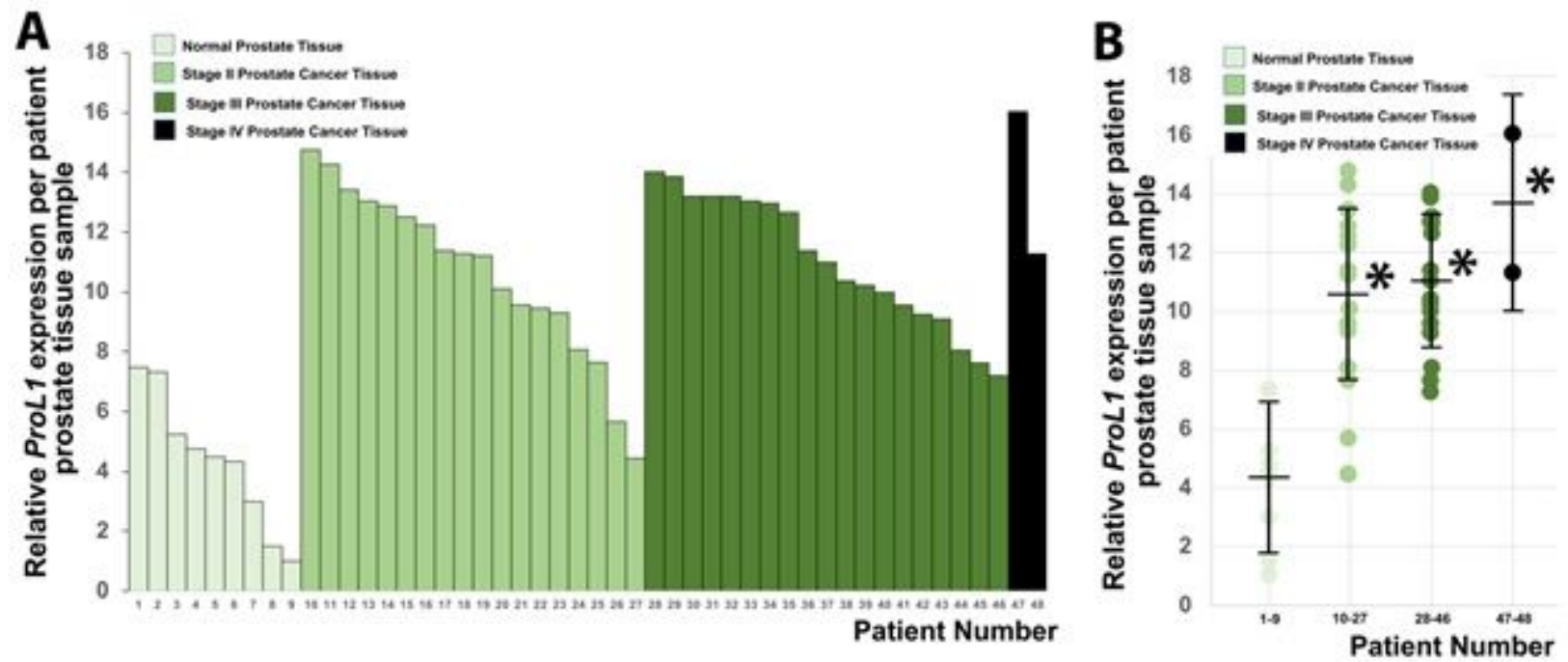
Figure 1

ProL1, the gene encoding opiorphin is upregulated in PrCa lines. A) Quantitative RT-PCR was used to determine relative expression levels of ProL 1 in a tissue CDNA microarray with sample from control and different stages of PrCa. B) The data for individual samples was normalized to the sample with the lowest level of ProL1 expression in the tissue array. Columns represent the average ProL1 expression level for each grade of cancer. There was significantly higher expression levels of ProL1 in PrCa tissue, with a trend for higher levels of expression as the cancer stage increased. Error bars $=$ Std. Dev., ${ }^{*}=p-$ value $<0.05$.
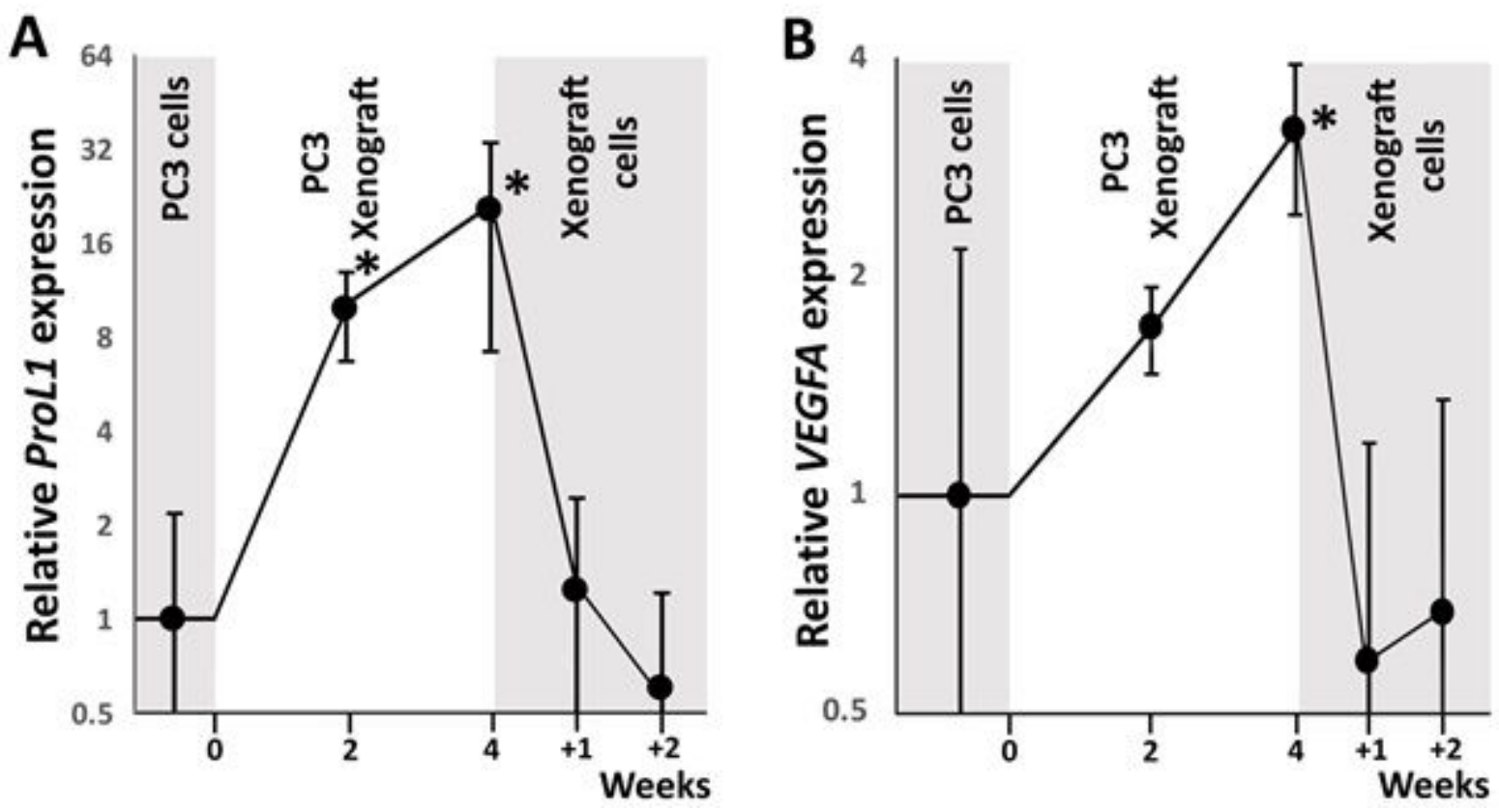

Figure 2

Relative expression of ProL1 and VEGFA in the PC3 PrCa cell-line before xenografting into nude male mice, in the xenografted tumors, and in cells isolated from the tumors and kept under normoxic cell culture conditions. At 2-weeks and 4-weeks post-xenograft animals were euthanized, tumors excised, and tumor tissue was used to isolate cells for cell culture. RNA was extracted from cells and tissues to perform qt-RT-PCR for the ProL1 and VEGFA genes, with expression normalized to the house-keeping gene, RPL19. The relative change in expression of A) ProL1 and B) VEGFA is represented as fold-change compared to the starting population of the parent PC3 cell-line ( $N=5$, error bars $=95 \%$ Confidence Interval, *= p-value $<0.05)$.

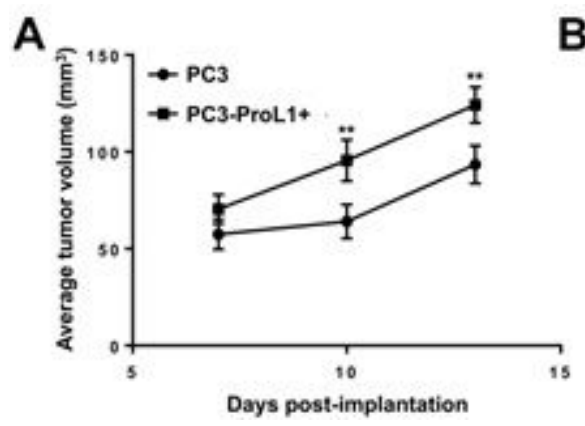

Days post-implantation
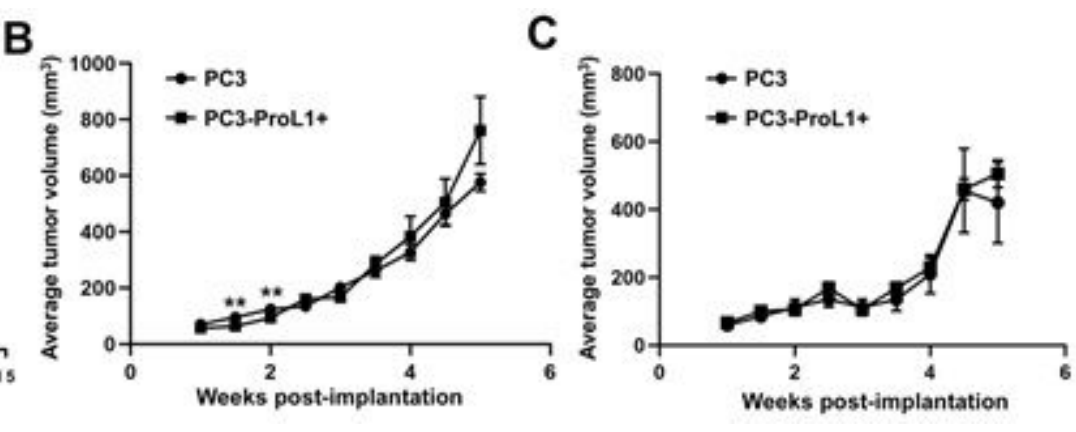


\section{Figure 3}

PC3 cells engineered to overexpress ProL1 have initially faster tumor growth in male mice compared to the parent cell-line. PC3 and PC3-ProL1+ cell-lines were injected into male and female nude mice and tumor size determined by standard caliper measurements ( $\mathrm{N}=10$ per group). All mice developed tumors. The average tumor volume is shown for A) male mice at early time points, B) all time points for male mice and C) all time points for female mice. Error bars $=$ Std. error * $=p$-value $<0.05$.
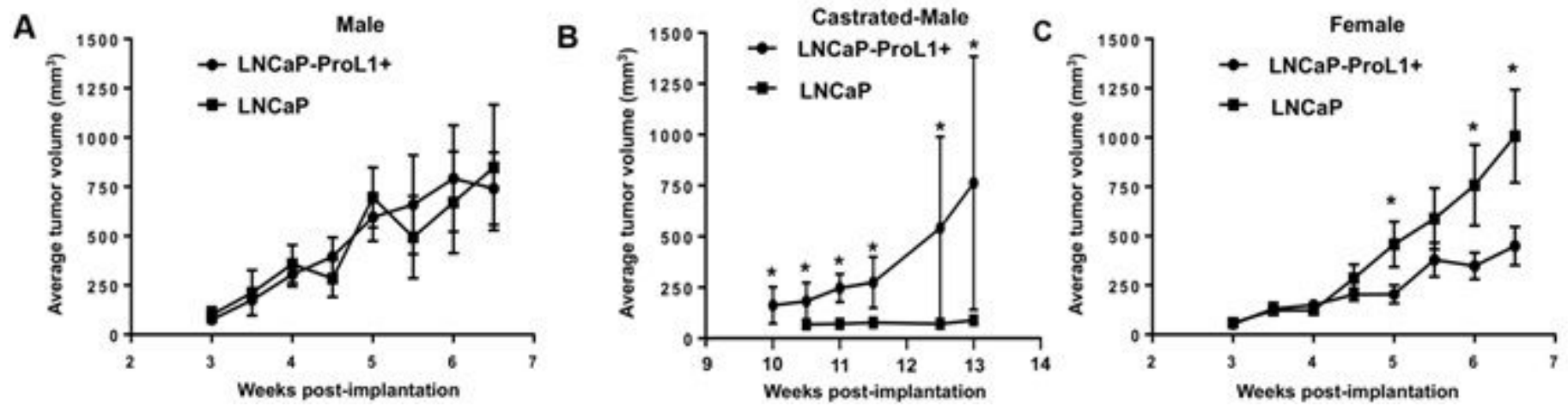

\section{Figure 4}

Growth of xenografted tumors derived from LNCaP-ProL1+ or LNCaP cell-lines in male, castrated male or female mice. LNCaP and LNCaP-ProL1+ cell-lines were injected into male, castrated-male and female nude mice and tumor size determined by standard caliper measurements. The average tumor volume is shown for each time-point. Error bars $=$ Std. Error. ${ }^{*} p$-value $<0.1$, ${ }^{*} p$-value $<0.05$. A) Growth of LNCaP or LNCaP-ProL1 derived tumors in male mice ( $N=10$ per group, all mice developed tumors). B) Growth of LNCaP or LNCaP-ProL1 LNCaP-ProL $1+$ derived tumors in castrated male mice ( $\mathrm{N}=5$ per group, 2/5 mice developed tumors in the LNCaP-ProL1+ group and 0/5 developed tumor in LNCaP group) C) Growth of LNCaP or LNCaP-ProL1+ derived tumors in female mice ( $\mathrm{N}=10$ per group, all mice developed tumor).

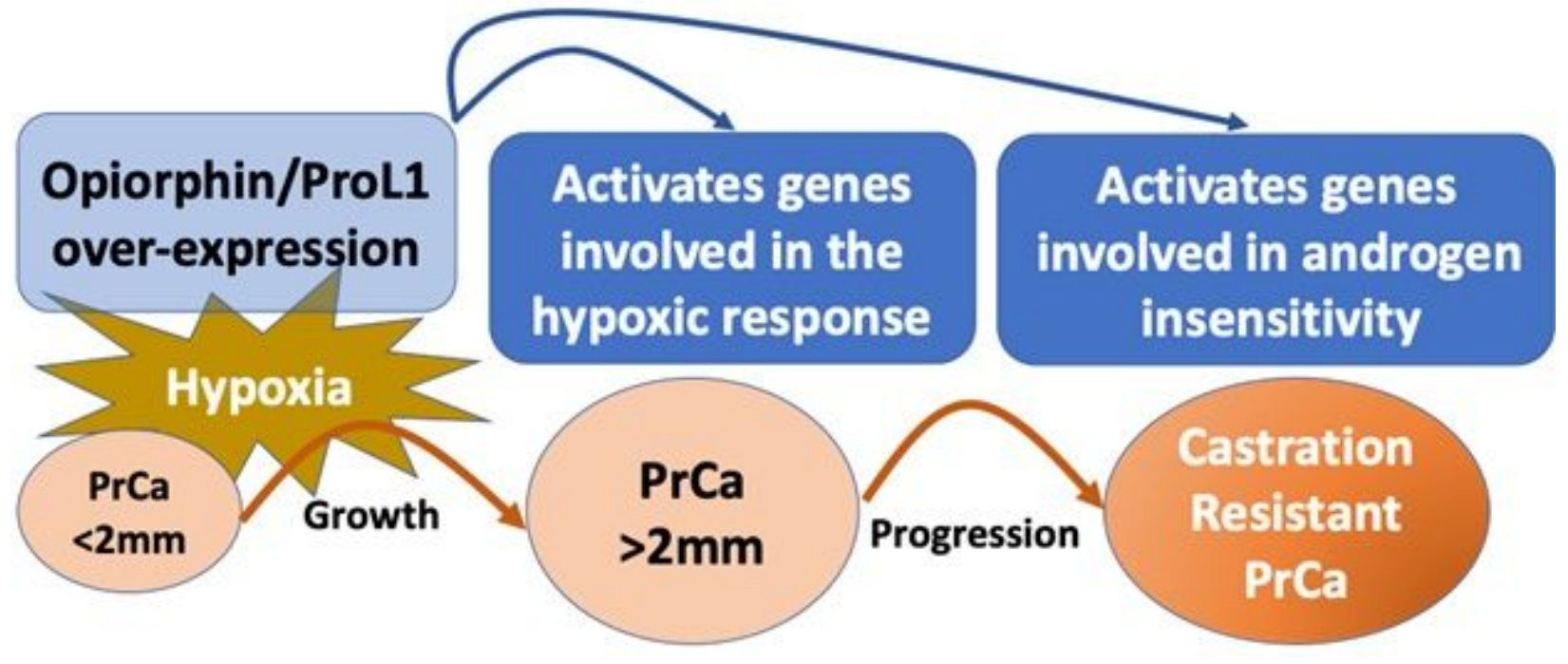

Figure 5 
In our recent publication we demonstrated that in corporal smooth muscle cells opiorphin is a master regulator of the hypoxic response. Therefore, we hypothesize that as the tumor grows above $1-2 \mathrm{~mm}$ hypoxia first causes up-regulation of opiorphin, which in turn activates expression of genes involved in the hypoxic response and the development of androgen-insensitive/ castration resistant PrCa.

\section{Supplementary Files}

This is a list of supplementary files associated with this preprint. Click to download.

- SupplementalTable1.csv

- SupplementalTable2.xIsx

- SupplementalTable3.docx

- SupplementalTable4.xlsx

- SupplementalTable5.xlsx

- SupplementalTable6.xlsx

- SupplementalTable7.xlsx

- SupplementalTable8.xIsx

- SupplementalTable9.xlsx

- BMCresubmitNC3RsARRIVEGuidelinesChecklistfillable.pdf 